\title{
Diabetic Ketoacidosis
}

National Cancer Institute

\section{Source}

National Cancer Institute. Diabetic Ketoacidosis. NCI Thesaurus. Code C50530.

The metabolic condition resulted from uncontrolled diabetes mellitus, in which the shift of acid-base status of the body toward the acid side because of loss of base or retention of acids other than carbonic acid is accompanied by the accumulation of ketone bodies in body tissues and fluids. 